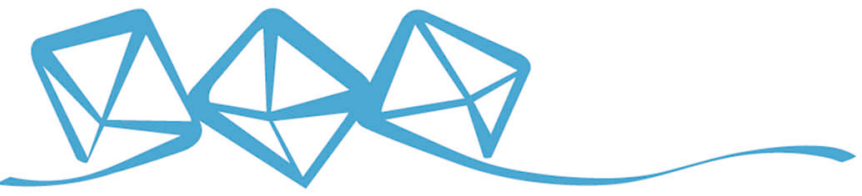 COMMUNICATIONS MATERIALS
}

ARTICLE

https://doi.org/10.1038/s43246-020-0016-3

OPEN

\section{Simulating nephron ion transport function using activated wafer electrodeionization}

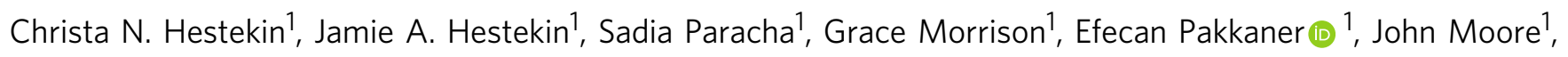
Leticia Santos de Souza ${ }^{1}$, Sam Stephens (1) ${ }^{1}$, Catey Atchley ${ }^{1} \&$ Ira Kurtz ${ }^{2,3 凶}$

Current approaches for treating patients with end stage renal disease include hemodialysis and peritoneal dialysis, both of which are diffusion-based treatments that require a dialysate solution. The native kidney has separate filtration (glomerulus) and transport (nephron) capabilities. Although artificial methods for simulating glomerular function using membrane ultrafiltration have been proposed, there are no known technologies for simulating the ion transport functions of the nephron. Here we have created a synthetic nephron using activated wafer electrodeionization (AWEDI). AWEDI incorporates mesh electrodes within an electrodeionization wafer, allowing ion selectivity and transport rate to be modulated independently. We demonstrate the capabilities of AWEDI with several physiologically relevant ions which mimic the fine control of ion transport by the kidney. Furthermore, by integrating our AWEDI technology with ultrafiltration, nanofiltration, and reverse osmosis modules, the possibility of incorporating AWEDI technology in an artificial kidney is demonstrated.

\footnotetext{
${ }^{1}$ Ralph E. Martin Department of Chemical Engineering, University of Arkansas, 3202 Bell Engineering Center, Fayetteville, AR 72701, USA. ${ }^{2}$ Department of Medicine, Division of Nephrology, David Geffen School of Medicine, University of California, Los Angeles, CA 90095, USA. ${ }^{3}$ Brain Research Institute,

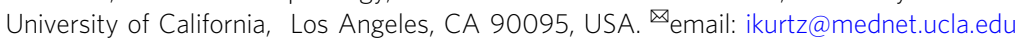


E ach year of the $\sim 47$ million patients in the US with chronic kidney disease (CKD), 100,000 develop end stage renal disease (ESRD) requiring that they receive some form of renal replacement therapy to remain alive ${ }^{1-4}$. There are currently $\sim 700,000$ ESRD patients who are being treated with hemodialysis (HD), peritoneal dialysis (PD), or renal transplantation ${ }^{1-4}$. Of those patients receiving dialysis, $\sim 100,000$ are on the kidney transplant waiting $\operatorname{list}^{5}$. The average duration of patient survival on dialysis is only 7.2 years with a 10 -year waiting time for a cadaveric donor kidney in large programs ${ }^{6-8}$. Approximately 100,000 patients die yearly on dialysis while waiting for a kidney transplant ${ }^{6-8}$.

It is generally accepted that $\mathrm{HD}$ and $\mathrm{PD}$ as intermittent modalities that are inherently non-physiologic are associated with significantly higher hospitalization rates and mortality compared to kidney transplant recipients ${ }^{8}$. Other than research into miniaturization and dialysate regeneration the basic technology used to treat patients with $\mathrm{HD}$ and $\mathrm{PD}$ has remained essentially unchanged for 40 years $^{9-12}$.

The kidney can be considered a "chemical computer" whose function is to sense and maintain the chemistry of blood relatively constant despite fluctuations due to changes in dietary intake. The kidney accomplishes this task by utilizing two separate fundamental processes. Initially, filtered blood is created by the glomeruli in a process that allows substrates smaller than $\sim 7 \mathrm{kDa}$ to freely permeate through the glomerular barrier ${ }^{13,14}$. This barrier prevents the cell components of blood and protein (albumin and globulins) from being excreted from the body. In the next phase the filtrate generated by the glomeruli flows into the nephron network (about a million nephrons per kidney), where specific transport processes transfer ions and organic molecules between the luminal fluid and peritubular blood thereby generating the final urine which is ultimately excreted ${ }^{15}$.

Attempts have been made to simulate the filtration function of the glomerulus. Nissenson et al. reported the possibility of simulating glomerular filtration (as well as with the ion transport functions of the nephron) using a pore-based nanofilter (NF) system, however; only theoretical modeling of its properties were reported $^{16}$. Uesaka described a nanostructure HD membrane made from polysulphone ${ }^{17}$ and Kanno et al. extended this work and described a porous polyethersulfone (PES) membrane with nanopores, which is predicted to have the same clearance of urea as the native kidney at $40 \%$ of the size ${ }^{18}$. Lee and Choi reported a microchannel-based system that aims to separate albumin protein from urea and glucose without using any membranes ${ }^{19}$. The microchannels were fabricated with soft lithography technology. The master was formulated using SU-8 2050 (a permanent epoxynegative photoresist) on a silicon wafer. Microchannels with $50 \mu \mathrm{m}$ depth were then fabricated using a polydimethylsiloxane polymer and then bonded to glass. The clearance of urea was $0.33 \mu \mathrm{L} / \mathrm{min}$ for a $1 \mu \mathrm{L} / \mathrm{min}$ flow rate and the experimental results were verified with numerical simulations as well. However, it was concluded that in order to reach the human kidney capacity with the fabricated device, almost 75,000 units needed to be integrated. Kensinger et al. have reported the development and testing of a silicon hemofilter cartridge that operates continuously without an external power or pumping source ${ }^{20}$. The filtration volumes were found to be clinically relevant, and no coagulation was observed in the device. However, the albumin levels in the filtrate were higher than the predicted values based on previous in vitro experiments. Moreover, the hemofilters faced mechanical failure after several days of implantation and the tests were terminated.

To simulate the transport capabilities of the nephron, various groups have proposed the use of living cell-based systems to accomplish its various transport requirements ${ }^{21}$ or stem cells that can repopulate glomerular, tubular, and vascular cells in decellularized kidney scaffolds 22 . A major challenge with these approaches are the known changes in cell transcript/protein expression when cells are removed from their native in vivo environment and utilized either as primary cells or immortalized $^{23,24}$. Another challenge includes the need for cultured tubular cells to maintain apical-basolateral polarity given the requirement of cells in the nephron to mediate vectorial transport $^{25}$. The ability of the nephron to modulate the transport of various ions and water independently in response to dietary changes is accomplished by multiple transport proteins expressed in multiple cells types that utilize physical, neurogenic, and hormonal signaling that is a challenge to replicate in vitro ${ }^{26}$. Thus far, these systems do not replicate the multiple transport properties of the nephron.

In this work, a new technology is demonstrated that can for the first time simulate certain ion transport functions of the nephron. This technology, of activated wafer electrodeionization (AWEDI), uses ion-wafer interactions followed by ionic transport through a membrane using an electric field as the driving force. In this completely artificial approach, this new technology does not involve any biological components. AWEDI utilizes highly permeable mesh electrodes allowing the transport chambers within the device to be turned on and off electrically. This property enables the independent modulation of both the specificity and rate of ion transport. The capabilities of AWEDI are demonstrated using several physiologically relevant ions to simulate the function of the nephron.

\section{Results}

Design and Working Principle of the AWEDI. The primary function of the nephron is to transport various cations, anions, organics, and water between the glomerular ultrafiltrate and peritubular blood ${ }^{15,26}$. The transport and excretion of each substance in the urine is in general modulated independently, and approximates the net input into the circulation from the diet. As shown in Fig. 1, AWEDI has similar capabilities. The kidney (panel a) uses specific transport proteins to selectively transport various ions and organics out of and into the nephron. Each transport process is localized to very specific cell types and sites along the nephron. The AWEDI uses a combination of ion exchange wafers (panel b) with differing ion selectivities. Flow comes into the system and ions are moved from the dilute (stream D) chambers to the concentrate (stream C) chambers by applying a voltage over electrodes 1 and 6 . The current drives the movement of ions as indicated by the inset with cations moving to the cathode (right) and anions moving to the anode (left). Cation (such as $\mathrm{Na}^{+}$) are prevented from leaving the concentrate chamber due to the presence of a membrane that only exchanges anions (AEM) and similarly anions (such as $\mathrm{Cl}^{-}$) are prevented from leaving the other concentrate chamber due to the presence of a membrane that only exchanges cations (CEM). In addition to just driving transport, the applied voltage also leads to water splitting and hydrogen gas production as indicated by the two reactions at the anode and cathode ends of the system. Ion selectivity is determined by the type of resin used in the AWEDI wafers (Supplementary Tables 1 and 2). The resins vary in their chemistry and bead size (i.e. surface area). The two features allow for different affinities and transport rates for the various ions. Using different ion exchange wafers and changing the electrodes (E1-E6) over which the potential difference exists, it is possible to change the selectivity of the AWEDI from $\left(\mathrm{Na}^{+}, \mathrm{K}^{+}, \mathrm{Ca}^{2+}\right.$, or $\mathrm{Mg}^{2+}$ selective). Shown is the relative removal of specific ions over a 24-h period using four different wafers with different ion selectivity (panel c). The data is shown primarily in terms of the 
a

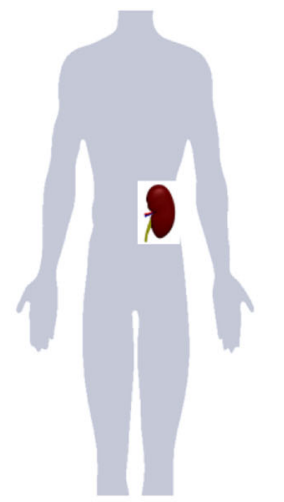

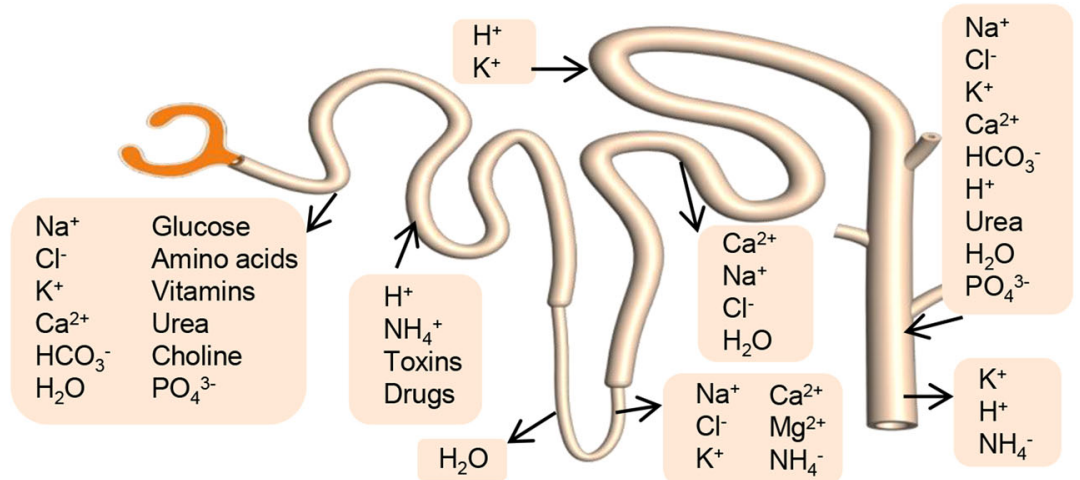

b

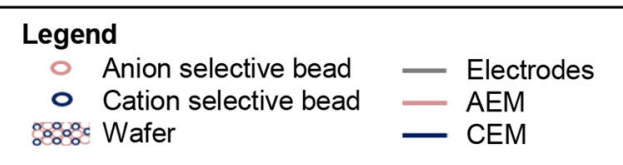

$2 \mathrm{H}_{2} \mathrm{O}->\mathrm{O}_{2}+4 \mathrm{H}^{+}+4 \mathrm{e}^{-} \leftarrow$

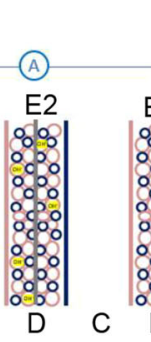

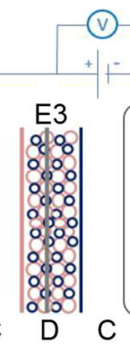
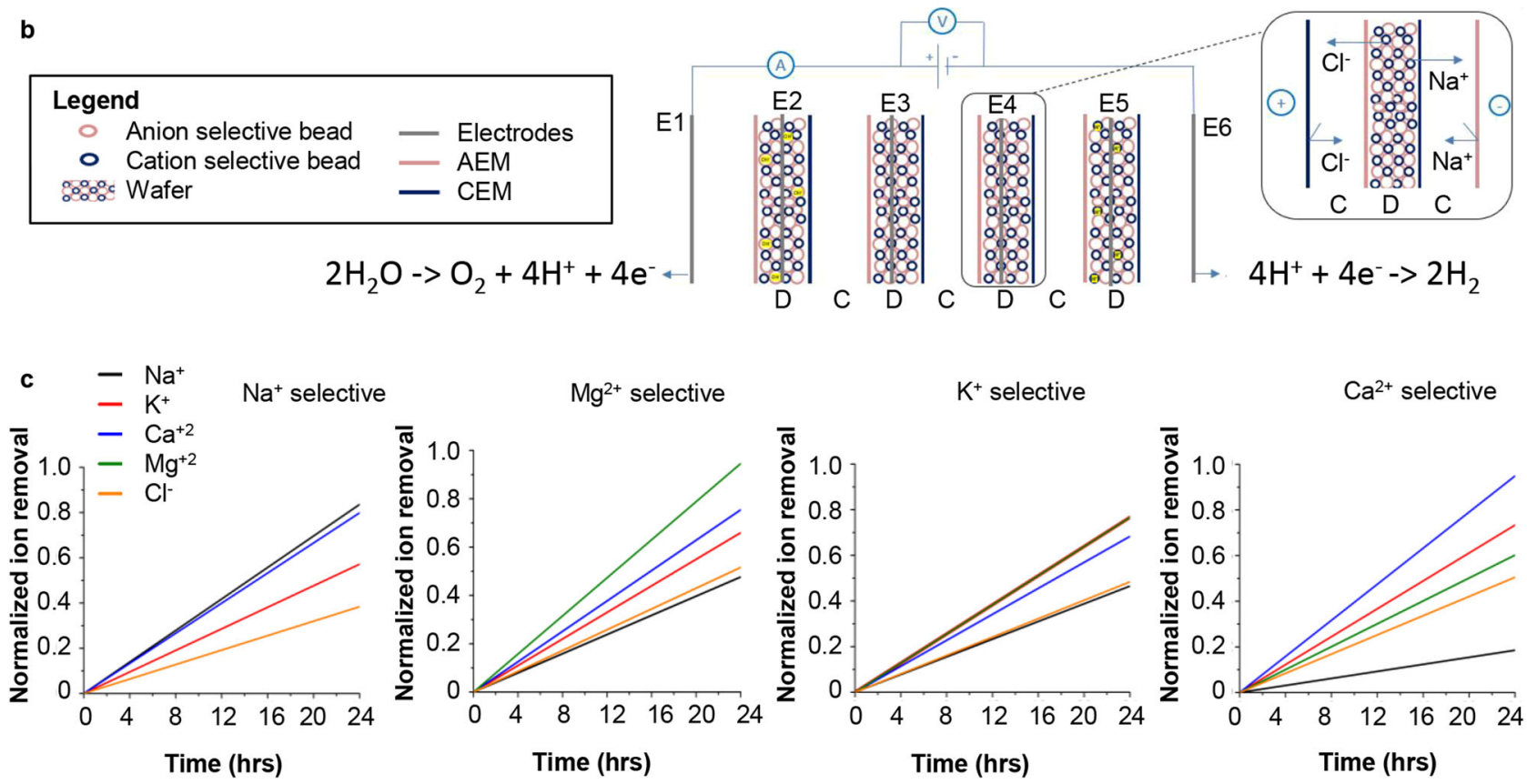

Fig. 1 Design and working principle of the activated wafer electrodeionization (AWEDI) for nephron simulation. a Transport processes in the nephron, b schematic of AWEDI wafer system, where C is concentrate stream, D is dilute stream, E1-E6 represent the six different electrodes for controlling ion selectivity, c data for different AWEDI wafers demonstrating high selectivity for different cations (sodium, potassium, calcium, and magnesium).

positive ions, since the transport of the counter-ion $\left(\mathrm{Cl}^{-}\right)$will be controlled by electroneutrality rather than selectivity. The low $\mathrm{pH}$ of the artificial urine stream eliminates significant bicarbonate excretion. The protons combine with bicarbonate and generate $\mathrm{CO}_{2}$, which is transferred from the synthetic urine stream into the stream returning to the blood. In this way bicarbonate is effectively absorbed from the synthetic urine stream to the blood return stream. Phosphate excretion is expected to be high since it is a trivalent ion and the EDI wafer will be highly selective for this ion. Since the flow will be split with a portion going to each wafer in the AWEDI, the total selectivity will be based on a combination of the individual wafer selectivities. Furthermore, AWEDI has an additional functional capability over traditional electrodeionization making it ideal for simulating nephron ion transport function. Specifically, AWEDI has an electrode in each wafer (panel b) which allows by a simple change of current the transport properties to be altered. When the circuit is connected between the electrode in the potassium wafer and the electrode on the anode of the system, only the sodium and magnesium selective wafers would be functionally active and thus the selectivity of this system would change (panel c). The potassium selective wafer in this instance is not active because there will be no membrane diffusion after wafer transport, since the electrode is located in the potassium electrode. In theory, it is possible to get individual transport of ions through each of the wafers using the electrodes positioned in the wafers. For instance, if it was desired that only the potassium wafer be active the energized electrodes would be the ones in the magnesium wafer electrode and calcium wafer electrode. Thus, this novel function of AWEDI enables a wide range of ion transport specificities that can be changed using only current, not flow. The transport of the ions is most often limited by the power density which is typically $25-35 \mathrm{~mA} / \mathrm{cm}^{2}$ for an EDI membrane ${ }^{27}$. Using Faraday's law of electrolysis and assuming only $50 \%$ efficiency for the transport of ions, this means that a membrane area of around $70 \mathrm{~cm}^{2}$ is required to transport the same amount of ions as the kidney does for urine production. We have also tested the variation of wafer 1 in multiple batches over 2 years and the variability in ion selectivity varies from $32 \%$ to $42 \%$ for the different ions. Although this variability may seem high, it includes a number of variables that would be further evaluated before using them in a medical device. These variables include variability of bead sizes, age of wafers, and varying concentrations of feed solutions. In fact, the variability of selectivity for a specific ion from run to run can range from $3 \%$ to $30 \%$ depending on the run concentrations, $\mathrm{pH}$, etc. It is expected that with future studies on the effects of these 

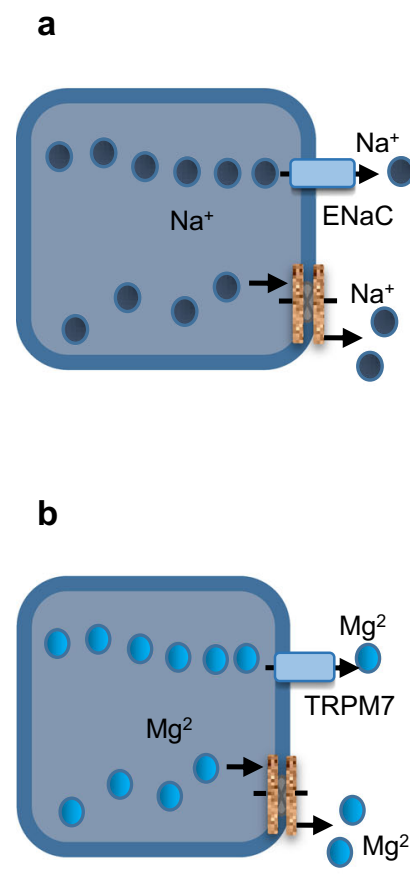
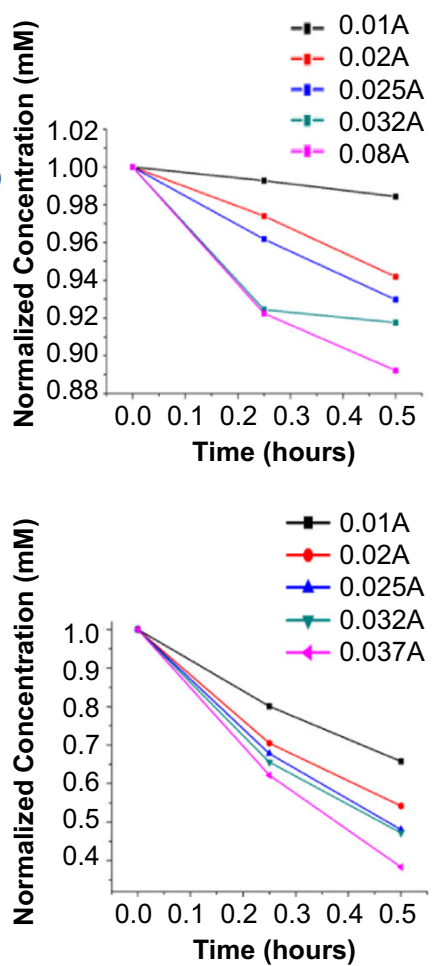
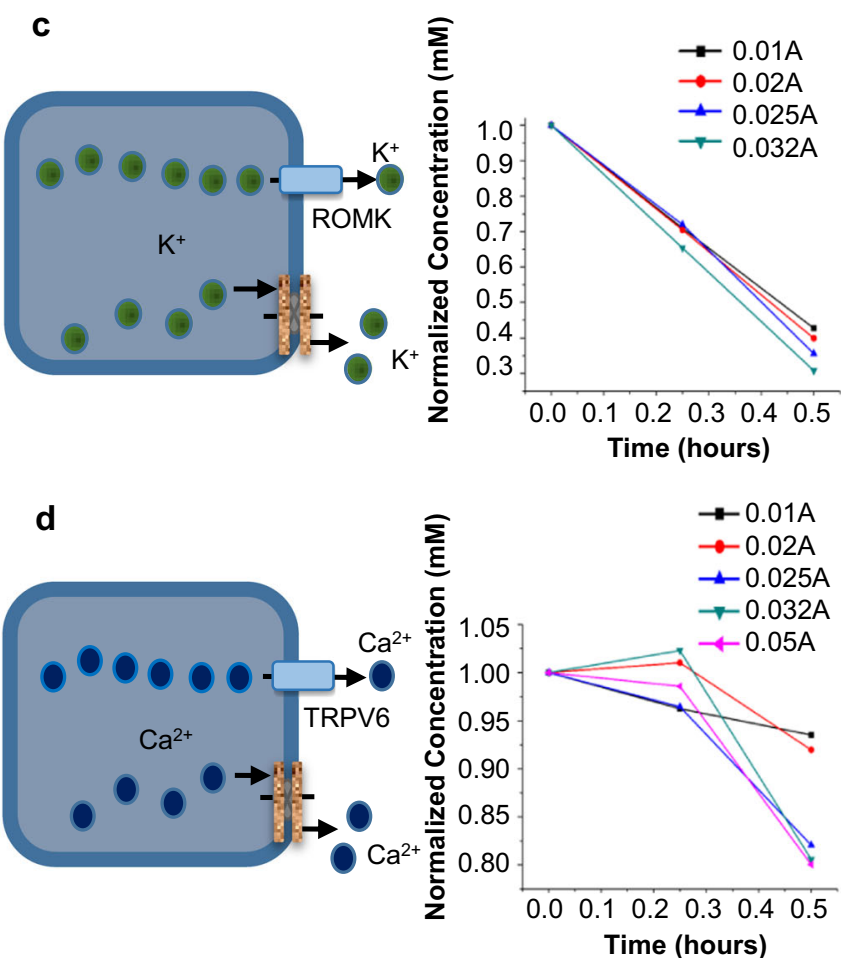

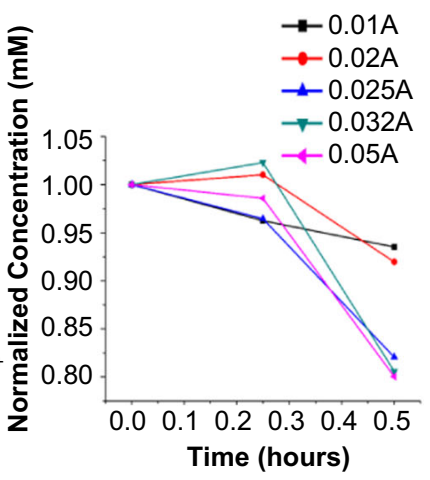

Fig. 2 Nephron ion transport compared to AWEDI. Ion transport processes in the nephron as examples that compare to the ability of AWEDI devices to specially modulate the transport of various ions at different applied currents: a sodium, b potassium, c calcium, and d magnesium.

variables that the run and batch variabilities can be significantly reduced.

Selective AWEDI Ion Transport Rates. Figure 2 shows an analogy between AWEDI transport function and the corresponding native kidney nephron transporters that mediate a similar ion transport function. Selective removal rates for sodium (panel a: function of $\mathrm{ENaC}$ channel), potassium (panel b: function of ROMK channel), calcium (panel c: function of TRPV6 channel), and magnesium (panel $\mathrm{d}$ : function of TRPM7 channel) are shown. The AWEDI also has the ability to control the rate of specific ion transport, similar to the nephron using current as the driving force. Response curves are shown for each ion at physiological concentrations. As shown, $>50 \%$ of the potassium and magnesium ions are removed in $30 \mathrm{~min}$ with higher currents leading to greater ion removal rates. The removal for sodium $(<10 \%)$ and calcium $(<20 \%)$ are lower in this time period because wafer 1 has a lower selectivity for these ions. Depending on the current used, all ions could be removed from a simulated blood solution in a period of 10-30 min using an AWEDI device with $950 \mathrm{~mL}$ total volume. The overall transport rate of each ion also depends on the flow rate of the ultrafiltrate and the size of the AWEDI device. In addition to the wafers shown here there have been other wafer experiments performed (Supplementary Figs. 1-6) as well as other combinations of wafers (Supplementary Figs. 7-9; Supplementary Table 3). In addition to changing the wafers, changing the current also allows the selectivity to be altered (Supplementary Figs. 7-9; Supplementary Table 3), which can be used to maintain an ideal concentration of ions, such as potassium in blood particularly following an acute increase in concentration (Supplementary Fig. 10). These figures show how changing the wafers and currents in the AWEDI change the selectivity of the overall system. While the studies thus far have focused on cation transport, the ability to transport both bicarbonate and phosphate have also been documented (Supplementary Figs. 11 and 12).

Physical and Chemical Properties of AWEDI Wafers. The physical and chemical properties of the AWEDI wafers have been characterized in Fig. 3. Panel a shows the microscopic characterization of the wafers before (virgin) and after (veteran) experiments using both SEM and optical microscopy. In the scanning electron microscopy (SEM) images it can be seen that the polymer is forming a loose connection to the bead material and thus allows durability without loss of functionality. While the polymer forms a loose enough connection to allow access to the ion exchange bead, there is no indication that the beads are being released over time. In a 6-month study, Potts et al. used EDI wafers in a fermentation broth with little to no change in performance over time, which suggest that little or no particles were released. Furthermore, visual inspection of the aged wafers did not show any particle release except in the case of exposure to highly acidic material such as glacial acetic acid ${ }^{28}$. The optical microscopy data shows the new feature of the AWEDI system which is the porous electrode inside of the wafer. As shown, the electrode does little to interfere with the overall structure of the wafer and thus, as seen in the results, the AWEDI wafers operate similarly to traditional ion exchange wafers. Panel b shows the Fourier-transform infrared spectroscopy (FTIR) of all the materials making up wafer and scans of the wafer after operation. As seen, the wafer material is a combination of the polymer as well as specific ion groups for $\mathrm{C}-\mathrm{N}$ stretching, $\mathrm{C}-\mathrm{C}$ stretching, and other identified functional groups. Interestingly, $\mathrm{Mg}-\mathrm{O}$ stretching is seen in the more magnesium selective wafer (wafer 9) but not the less magnesium selective wafer (wafer 1). This qualitatively indicates that the wafer taking up more magnesium is one of the reasons for the increased selectivity in the 

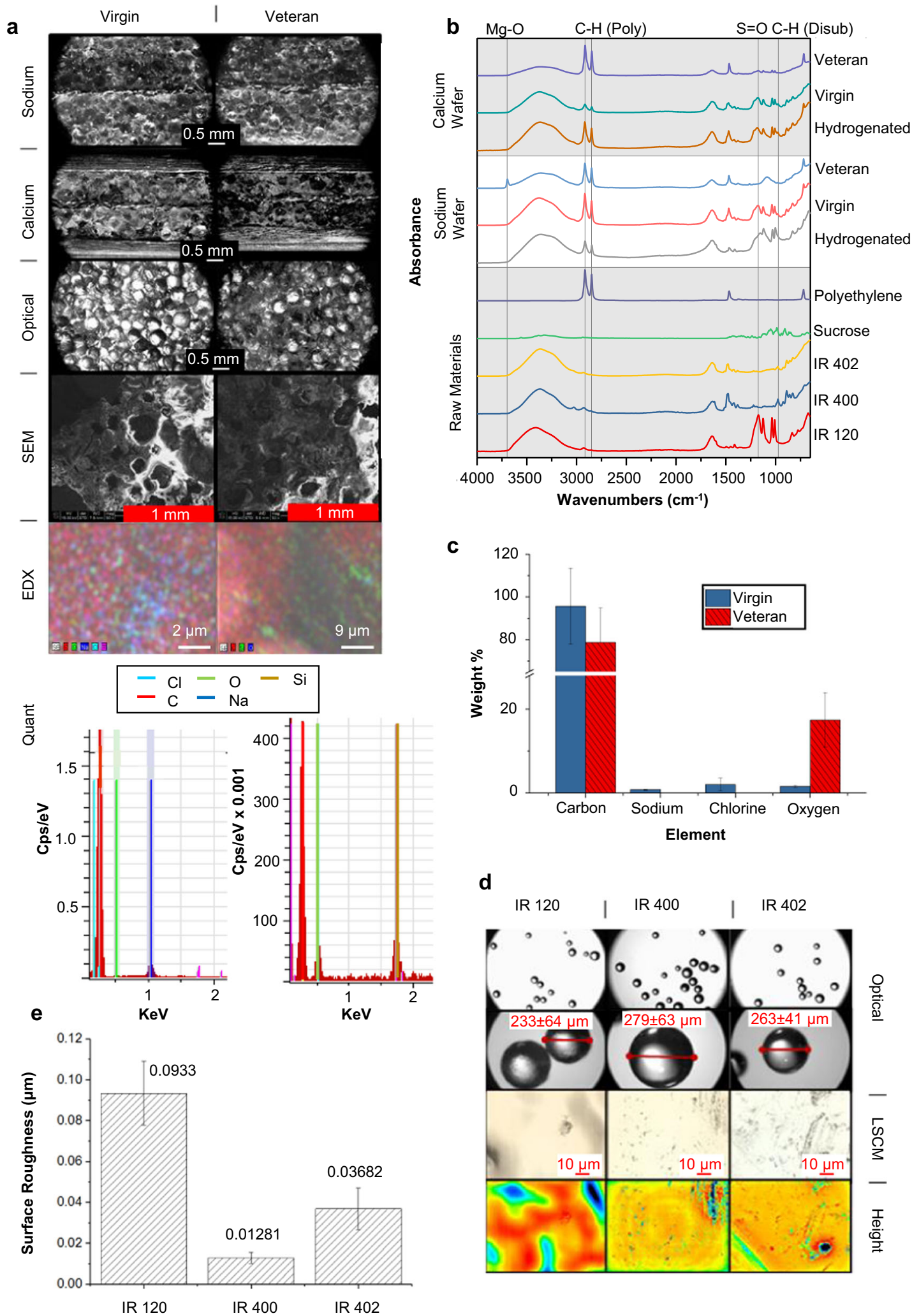

Element

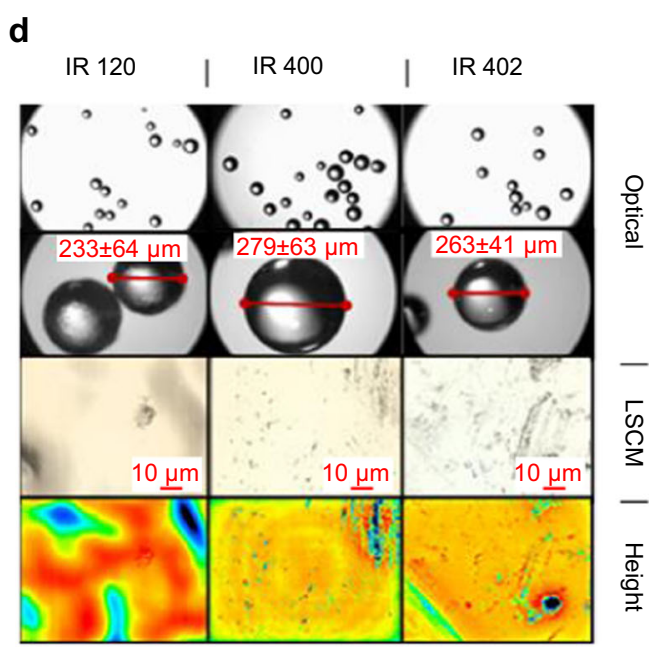

Fig. 3 Advanced material chemical and image analysis for AWEDI wafers. a Optical, scanning electron microscopy (SEM), energy dispersive X-ray analyzer (EDX), and quant graph images for a sodium selective vs. calcium selective wafer, b Fourier-transform infrared spectroscopy (FTIR) analysis of AWEDI wafers before (virgin) and after (veteran) an experiment, $\mathbf{c}$ chemical composition (mean \pm standard deviation) of the AWEDI wafers before (virgin) and after (veteran) an experiments based on the EDX images, $\mathbf{d}$ optical and 3-D laser scanning confocal microscope (LSCM) images of the resin beads that make up the AWEDI wafers with their respected height map, and e surface roughness of the beads (mean \pm standard deviation). 
Mg selective wafer. Panel c shows scanning electron microscopyenergy dispersive X-ray analyzer (SEM-EDX) mapping of the wafers. In the wafer shown (Na selective wafer), it is seen that the wafer before the run has a significant amount of sodium. After running for several cycles, the sodium decreases (indicated by decrease in the amount of blue in the figure) indicating that the wafer is self-regenerating and thus the majority of the active sites are bonded with hydrogen which are not seen in EDX. The increase in oxygen is evidence of hydroxyl's attached to the anion exchangers; further evidence that water splitting is driving the transport. The counter anion that goes toward the anode is hydroxide or the other counter ions in blood such as chloride or phosphate. In the AWEDI system, hydrogen will be made at the anode and oxygen at the cathode. However, both of these gases are in very small amounts and will leave with the synthetic urine stream. Panel d shows the surface properties of the beads using confocal microscopy along with showing the characterization of the AWEDI wafers using surface roughness analysis. The beads form a heterogeneous structure due to their non-uniformity; also shown is heat mapping for surface roughness. The surface roughness of the AWEDI wafers is calculated between 0.01 and 0.1 micron for the different ion exchange beads used in the AWEDI wafers. Other analyses of the AWEDI wafers are included in Supplementary Figs. 13-16 which include particle size analysis, other EDX, and other SEM pictures. A schematic for production of the wafers is also included in Supplementary Fig. 17.
Artificial Kidney Device. Given the capability of the AWEDI to transport specific ions like the nephron, a proof of concept series of experiments with the AWEDI incorporated into an artificial kidney device was performed (Fig. 4). Panel a shows a schematic representation of the components of the artificial kidney device. Initially, an ultrafiltration membrane prevents blood cells and proteins from being excreted in the artificial urine stream. The cells and proteins are returned to the blood stream. This is similar to how the glomerulus functions in the native kidney. Supplementary Figs. 18-20 show the long-term function and performance with blood of the ultrafiltration membrane. The next components in the device are two EDI units (a traditional EDI and an AWEDI) with a NF in between. The traditional EDI is used to modulate the rate of potassium transport into the artificial urine stream. A nanofiltration membrane is required to prevent glucose from significantly entering the artificial urine stream and accordingly return it to the blood stream. Several commercially available nanofiltration membranes were tested for their ability to retain glucose $(>75 \%$ rejection) while passing urea $(<20 \%$ rejection) as shown in Supplementary Table 4. Across the NF, the estimated osmotic pressure difference is 80 psig. The forward hydrostatic pressure gradient of 100 psig exceeds this. The size of various uremic toxins is such ${ }^{29}$ that they will not permeate the NF membrane. To increase the excretion of these substances, one possibility would be to remove the NF from the fluid stream under software control for a percentage of the total treatment time the device is functioning. However, during this time glucose
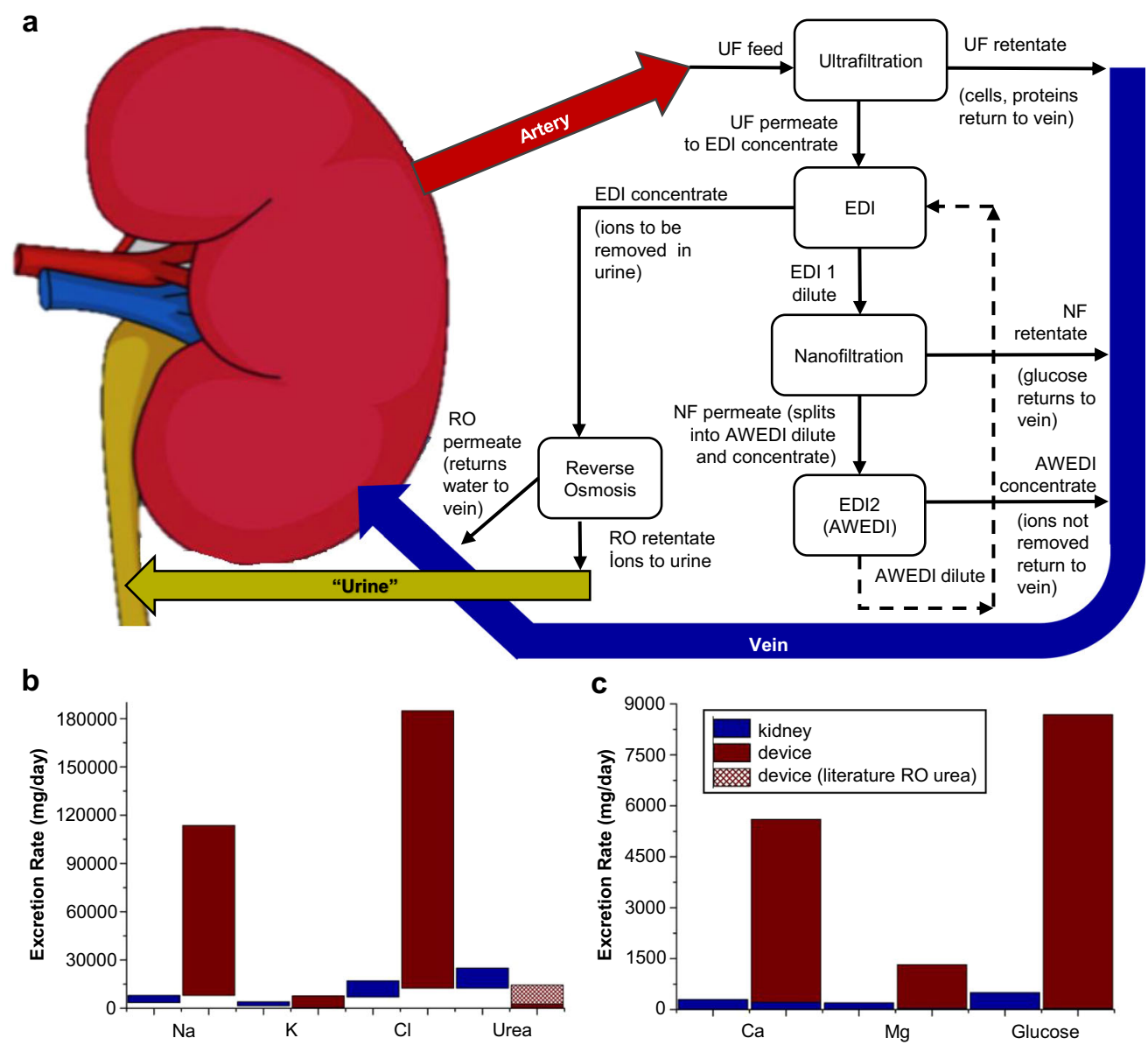

Fig. 4 Configuration and performance of the artificial kidney device. a Various components of the artificial kidney device. $\mathbf{b}$, c Comparison of the composition of urine produced by a native kidney (blue) to artificial urine produced by the artificial kidney device (red). Device performance was calculated using experimental rejection values for ultrafiltration (UF), nanofiltration (NF), and reverse osmosis (RO) and selectivity values for electrodeionization 1 (EDI1) and electrodeionization 2 (EDI2) under different experimental conditions (transmembrane pressure, applied currents, flowrates, etc.). 
excretion in the synthetic urine would increase. Finally, a reverse osmosis (RO) membrane was used to provide the capability of modulating the amount of water in the artificial urine stream and accordingly the amount of water in the ultrafiltrate that will be returned to the blood stream. The amount of water excreted in the synthetic urine (plus insensible water loss) ultimately will approximate the dietary water intake. In this way, the blood osmolality will be kept constant within a narrow range. Across the RO membrane the estimated osmotic pressure difference is 100 psig. The forward hydrostic pressure gradient is 120 psig, which is greater than the osmotic pressure gradient. Although commercially available RO membranes typically have very high rejections of ions, they are also commercially run at high pressures. The results shown in Supplementary Table 5 and Supplementary Fig. 21 were obtained at low pressures ( $<30$ psi) allowing the $\mathrm{RO}$ membrane to be run at low power and provide greater safety for devices that are ultimately implanted. Although these results show low urea rejection, there is literature to show that higher rates of urea rejection are possible ${ }^{30}$. Figure 4 panels $b$ and $c$ show the expected artificial urine stream excretion rate of a native kidney and the calculated excretion rate of the AWEDI device. As shown, the AWEDI device is currently able to transport each ion over a wider range of rates than the native kidney. Since the NF, AWEDI units and RO units will not be exposed to cells or significant amounts of protein, they have an intrinsic lifetime of years.

\section{Discussion}

It has been demonstrated for the first time the principles, design, and operation of a novel technology that simulates the physiologic transport of ions by the nephron. The AWEDI wafers were designed utilizing known physiologic lessons of how the nephron accomplishes many of its transport functions without replicating nephron function exactly. These principles include the requirement for substrate specificity and transport rate modulation based on changes in blood chemistry that result from fluctuating dietary intake. Compared to the nephron our system is currently not as selective. The selectivity of the AWEDI systems for the various ions is currently between 0.5 and 23 (Supplementary Table 2) as compared to sodium (Fig. 1 panel $\mathrm{d}$ and Supplementary Figs. 1-6). The results in Fig. 4 demonstrate that this selectivity profile allows excretion rates that cover the excretion range of the native kidney. It is possible to increase the selectivity profile of the AWEDI using binders that are more selective than conventional ion exchange beads. Ruckh et al. used quantum dots to make sensors with very high selectivity for potassium ${ }^{31}$. Another material used to increase ion selectivity is polyamidoamine dendrites to increase the sodium permeability of living neuronal cells $^{32}$, and to develop a fluorescent chemosensor with fluoride ion selectivity ${ }^{33}$. Zeolites are also recognized as useful to increase ion selectivity, where zeolite was incorporated into a porous membrane leading to vanadium ion and proton separation ${ }^{34}$. In the membrane fabrication field, Hosseini et al. found iron-nickel oxide nanoparticles contributed to a permeability increase for bivalents ions ${ }^{35}$. All of these approaches can be potentially utilized to improve the selectivity of the AWEDI units.

The wafers were made of polyethylene, as a binder, ion exchange material, and a porous electrode. Sugar is a space filler which dissolves away creating porosity. Polyethylene's polymerized C-H stretch (2950-2800) is evident in the final material, while sugar moieties are not present in the final structure. IR 120 has $\mathrm{S}=\mathrm{O}$ groups (1175) that are present in the final material (Fig. 2, panel b). This is the active functional group of the bead. As the $\mathrm{S}=\mathrm{O}$ bond opens and the sulfoxide accepts the contaminants, the amount of $\mathrm{S}=\mathrm{O}$ bond vibrations visible on the
FTIR spectra decreases. IR 400 and IR 402 are functionalized with trimethyl amine. Trimethyl amine when bound to an organic molecule can undergo further crosslinking between the methyl group on the amine and the aromatic ring structures present in the resin beads. Furthermore, crosslinking creates disubstituted $\mathrm{C}-\mathrm{H}$ groups (970) that are present only on IR 400/402. The main difference between the two resins is crosslinking time. For example, 402 is less crosslinked to allow for larger molecules to be able to interact with the surface chemistry, consequently the presence of disubstituted $\mathrm{C}-\mathrm{H}$ is reduced. As the material reaches the end of its life, it has donated all its excess hydrogen (including disubstituted $\mathrm{C}-\mathrm{H}$ ) and the $\mathrm{S}=\mathrm{O}$ active group have been reduced away. Mostly $\mathrm{C}-\mathrm{N}$ moieties dominate and $\mathrm{Mg}-\mathrm{O}$ stretching in the more magnesium selective wafer (sodium) begin to reveal themselves. The surface roughness measurements of the ion exchange beads that have been used to produce AWEDI wafers were carried out by optical microscopy. Previously, $\mathrm{Ng}$ et al. have reported that the surface roughness and porosity of biomaterials that are in contact with the blood stream could unfavorably effect the flow of blood by the entrapment of macromolecules ${ }^{36}$. Moreover, Umezu and co-workers have investigated the effect of surface roughness on the stability of red blood cells ${ }^{37}$. They have concluded that increasing the roughness from 0.45 to $2 \mu \mathrm{m}$ have caused a 10 -fold increase in the hemolysis rate. However, our measurements indicated that the bead with the highest roughness had a roughness value of $0.1 \mu \mathrm{m}$, therefore; it can be safely concluded that the wafers used in the AWEDI device could be stated as hemocompatible in terms of surface roughness. Moreover, the AWEDI and EDI are not in contact with blood per se but rather an ultrafiltrate.

Routing operations such as pump driving and wafer energization are managed by a microcontroller, while higher level operations including controls calculations and user interfacing can be handled by a small Linux-based computer in which current operating conditions can be monitored and controlled. The control system utilizes feedforward feedback controls to alter the concentration of ions that are sensed in the utrafiltrate and transported by the device. These concentrations are used to calculate feedforward responses according to modeling and experimental results. A proportional-integral-derivative controller (PID) provides feedback modulation to control for changes in ion concentration due to dietary intake. The system operates within predefined safety margins for each ion, where ion removal will stop if the concentration drops below a safe limit. The relative importance of removal rate for the varying ions is dictated by weighting factors in which an ion that is more critical to control accurately is given a higher factor. This is an area in which future improvements are possible. An interesting approach would be the application of machine-learning algorithms towards simultaneously controlling the several coupled outputs required for total ion control.

Our wafers can be compared to existing methodologies in the literature that can be divided into filtration methods and electrically driven methods. Current dialysis is based on passive transport using a concentration gradient between the blood and dialysate, which requires a dialysate and purified water infrastructure $^{38}$. In addition, filtration methods such as ultrafiltration, separate based on size of relatively large particles and are unable to modulate the differential excretion of small ions ${ }^{39}$. Nanofiltration could be used for a more selective separation of ions, however once the nanofiltration membrane is in place its selectivity cannot be easily changed unlike electrically driven systems where the current can be used to alter the selectivity as is needed in an artificial kidney device. Electrically driven methods include ion exchange, electrodialysis, and electrodeionization. Ion exchange has been shown to be used for selective separations as an effective 
method for separating ions from each other ${ }^{40}$. However, ion exchange requires regeneration which prevents it from being operated continuously and the loose beads are difficult to seal within the system. Electrodialysis is a continuous process which can be used for selective separations, however, these separations have limited chemistries because many of the chemistries used in beads cannot meet the requirements for forming membrane structures $^{41}$. Conventional electrodeionization utilizes ion exchange beads, with many different selective groups, to be used in the presence of electrodialysis providing a continuous process with the enhanced selectivity of ion exchange ${ }^{42}$. Conventional electrodeionization is not selective enough and has internal leaking problems of up to $2 \%$ per pass making it difficult to incorporate into a biomedical system. Therefore electrodeionization with AWEDI is seen as the only viable method for selectively transporting ions from blood that has been ultrafiltered that gives both the possibility of selective separation and changing selectivity depending on blood chemistry.

The data on the artificial kidney proof of concept experiments suggests that a modified device could ultimately function as a wearable and potentially implantable renal replacement device. Our technology could also be used in a standalone configuration or in conjunction with PD to control the chemistry of PD solutions such that less dialysate would be needed. Future wearable or implantable artificial kidneys using our technology will require biocompatibility analyses, miniaturization, control software development, and power utilization modification.

\section{Methods}

\section{Fabrication and operation of the AWEDI system. The AWEDI wafers were}

made by inserting a platinum porous meshes in-between two ion-exchange wafers. The meshes served as an electrode (anode or cathode) when a voltage was applied. In the absence of a voltage each mesh allowed the free passage of the feed solution. Controllable groups of meshes were connected to a power lead to allow the AWEDI to modulate the magnitude and specificity of ion flux by changing the applied voltage. In addition to the porous electrodes, the AWEDI wafer contained both anion and cation exchange resins, polymer (polyethylene powder, 500 micron), and sucrose. Seventeen different wafer combinations were tested using ion exchange beads thought to have selectivity of different ions including sodium, potassium, calcium, and magnesium. The polymer was used to bind the resins together, and sucrose was used to create porosity in the wafer.

A custom iron cast was constructed $(127 \mathrm{~cm} \times 127 \mathrm{~cm}$ diameter) for making the wafer. Each batch of wafer ingredients were mixed in a 23:23:15:10-g ratio of anion-resin:cation-resin:sugar:polymer. The composition was then mixed at a rate of $300 \mathrm{rpm}$ for $5 \mathrm{~s}$ using Flacktek Inc. SpeedMixer (model: DAC $150 \mathrm{SP}$ ) to enhance uniformity throughout. Wafer material was spread in cast, and then inserted into a Carver Press (model 3851-0), and run at 10,000 psi and $237^{\circ} \mathrm{F}$ for $90 \mathrm{~min}$, followed by a 20 -min cooling period via pressurized air treatment. After cooling, the cast was removed, and wafer carefully extracted. Each casting created 4-5 individual wafers for creation of the final AWEDI wafers. The wafer material was then soaked in di-water for at least $24 \mathrm{~h}$ allowing the sucrose to dissolve out creating the wafer pores.

To operate the AWEDI device, $400 \mathrm{~mL}$ of simulated blood containing $\mathrm{NaCl}$ $(5.6 \mathrm{~g} / \mathrm{L}), \mathrm{KCl}(0.29 \mathrm{~g} / \mathrm{L}), \mathrm{CaCl}_{2} \cdot 2 \mathrm{H}_{2} \mathrm{O}(0.34 \mathrm{~g} / \mathrm{L}), \mathrm{MgCl}_{2} \cdot 6 \mathrm{H}_{2} \mathrm{O}(0.02 \mathrm{~g} / \mathrm{L}), \mathrm{NaH}_{2} \mathrm{PO}_{4}$ $(0.08 \mathrm{~g} / \mathrm{L})$, urea $(0.1 \mathrm{~g} / \mathrm{L})$, creatinine- $\mathrm{HCl}(0.01 \mathrm{~g} / \mathrm{L})$, and $\mathrm{NaHCO}_{3}(2.1 \mathrm{~g} / \mathrm{L})$ was used. Streams of $\mathrm{NaCl}(20 \mathrm{~g} / \mathrm{L})$ and $0.3 \mathrm{M}$ sodium sulfate were used to concentrate chamber and rinse solution, respectively. The three solutions were pumped into the AWEDI cells at a rate of $250 \mathrm{ml} / \mathrm{min}$. Power was supplied by clamping positive and negative leads to the electrodes in a voltage range of $2-7 \mathrm{~V}$ set at a constant current of $0.02 \mathrm{~A}$. The EDI for the potassium removal is similar to the AWEDI except that no electrodes are inserted in the wafers. The rest of the production procedure is the same.

\section{Operation and fabrication of the ultrafiltration hollow fiber membrane. The} purpose of the UF is to return cells (RBC, WBC, platelets), proteins, and other large biological materials to the blood stream and prevent their excretion in the artificial urine stream. The UF was composed of hollow fiber membranes consisting of $\sim 17.8 \%$ polysulfone. The hollow fiber surfaces were modified with polydopamine $(0.3-0.5 \mathrm{mg} / \mathrm{mL})$ to decrease fouling. UF performance was assessed using rejection of BSA and IgG as well as monitoring flux decline. Typical rejection values were $>90 \%$ for both BSA $(91 \pm 8 \%, n=5, \leq 40$ psi) and $\operatorname{IgG}(92 \%, n=1, \leq 20 \mathrm{psi})$. During a 32 -day run of the UF system, the average BSA rejection was $79 \pm 12.5 \%$. In terms of flux, there was a significant drop in the first day (from 422.5 to $126.8 \mathrm{~mL} / \mathrm{min} \mathrm{m}^{2}$ ). In days 2 through 16 , the average flux was $72 \pm 48 \mathrm{~mL} / \mathrm{min} \mathrm{m}^{2}$.
Over the 32-day period, the flux was $\sim 140 \pm 145 \mathrm{~mL} / \mathrm{min}^{2}$. The hollow fibers were tested for potential tears within the membranes by embedding the fibers in water and running $\mathrm{N}_{2}$ gas through each fiber. The fibers with no leakage were placed in $100 \%$ ethanol for $6 \mathrm{~min}$ to $72 \mathrm{~h}$. Then the fibers were dried and the ultrafiltration unit was constructed using two elbows, a tee and connector tubing. The fibers in the elbow region were embedded in epoxy glue in order to manufacture a crossflow filtration and separate the feed, permeate, and retentate flows To decrease fouling, the membrane surfaces were modified using polydopamine (PDA). Before coating, $100 \%$ ethanol was used to wash the fibers for $30 \mathrm{~min}$ at 0 psig with pump dial speed of 2.5 . The fibers were then washed with di-water for $1-2 \mathrm{~h}$ at $0 \mathrm{psig}$ with pump dial speed of 2.5. Dopamine hydrochloride $(0.3-0.5 \mathrm{mg} / \mathrm{mL}$, Sigma Aldrich, St. Louis, MO) in $10 \mathrm{mM}$ Tris- $\mathrm{HCl}(\mathrm{pH}=8.6)$ solution was stirred at $25^{\circ} \mathrm{C}$ for $\sim 4-5 \mathrm{~h}$. The PDA coating was applied to the membranes for $3-5 \mathrm{~h}$ at 0 psig and pump dial speed of 2.5 . After coating the fibers, deionized water (di-water) was used at 0 psig for $1 \mathrm{~h}$ to wash away the excess PDA solution. In addition, some preparations also used a $50 \mathrm{mM} \mathrm{HCl}$ rinse at 0 psig for $20 \mathrm{~min}$. Next, air was pumped for 1-2 min and the unit was let to dry overnight. Each unit was tested initially using di-water at different pressures. Hematocrit adjusted whole bovine blood (Lampire Biological Labs, Pipersville, PA) was used to determine the membrane's rejection of blood cellular components (rejection: $100 \%$ RBC, 99.4\% WBC, 98\% proteins). In order to evaluate the membrane's rejection of blood proteins IgG (bovine, MP Biomedical, Santa Ana, CA) and BSA (Amresco, Solon, $\mathrm{OH}$ ) were tested. Solutions with $3.2 \mathrm{~g} / \mathrm{L}$ BSA or $0.75 \mathrm{~g} / \mathrm{L}$ IgG was prepared in PBS ( $\mathrm{pH} 7.4$ ) with $0.02 \%$ sodium azide. The experiments were conducted a pump dial speed of 2.5 at room temperature with a stirrer setting of 200 RPM. The retentate and feed pressure were in the range of 30-45 psig for both the BSA and IgG experiments. Flowrates were determined for both permeate and retentate streams by measuring the time taking to collect a desired sampling volume. At each time point, sample concentrations were determined using a NanoDrop spectrometer at $280 \mathrm{~nm}$

Nanofiltration membrane function. The purpose of the NF membrane was to prevent glucose from being excreted in the artificial urine stream and to return it to the blood stream. Two commercial NF membranes (NF-3a and NF270) were characterized to their ability to reject glucose while allowing the passage of urea. The NF-3a provided a reasonable rejection of glucose $(79 \pm 9 \%, n=3, \leq 60 \mathrm{psi})$ while still allowing the passage of urea $(86 \%$ passage $\pm 7.5 \%, n=3)$. The NF270 membrane provided the highest rejection of glucose $(98 \pm 1 \%, n=2, \leq 73 \mathrm{psi})$ but also had the lowest passage of urea $(4 \pm 4 \%, n=2)$. A Sterlitech HP4750 stirred cell was utilized with a membrane area was $19.63 \mathrm{~cm}^{2}$. The solution used to test the NF membrane was composed of $2.85 \mathrm{~g} / \mathrm{L}$ sodium, $0.16 \mathrm{~g} / \mathrm{L}$ potassium, $0.02 \mathrm{~g} / \mathrm{L}$ magnesium, $0.095 \mathrm{~g} / \mathrm{L}$ calcium, $3.72 \mathrm{~g} / \mathrm{L}$ chlorine, $0.10 \mathrm{~g} / \mathrm{L}$ urea, $0.01 \mathrm{~g} / \mathrm{L}$ creatinine, and $1.00 \mathrm{~g} / \mathrm{L}$ glucose. The solution (maximum $250 \mathrm{~mL}$ ) was stirred at $200 \mathrm{RPM}$ (room temperature) and pressurized with $\mathrm{N}_{2}$ gas at 30 psig. A DNS (3,5-dinitrosalicylic acid) assay was used to determine the rejection of glucose by the membrane, as described in the next section. A glucose rejection value of $98 \%$ was achieved.

Assays for sodium, potassium, calcium, magnesium, chloride, urea, glucose, and bicarbonate. Atomic absorption was utilized with an air-acetylene tank (40-45 psig air, 10-15 psig acetylene) to assay the concentration of sodium, potassium, calcium, and magnesium. For sodium, the samples were diluted $50 \times$ using di-water and the absorption wavelength was $330.2-330.4 \mathrm{~nm}$ with a lamp current of $5 \mathrm{~mA}$. For potassium, the sample was not diluted and the absorption wavelength was $404.4 \mathrm{~nm}$ with a lamp current of $6 \mathrm{~mA}$. For magnesium, 5-20 ppm standards were used without dilution with a lamp current of $3 \mathrm{~mA}$ with air-acetylene flame. The wavelength was $202.6 \mathrm{~nm}$ with a slit width of $1 \mathrm{~nm}$. For calcium, the samples were diluted $30 \times$ using di-water and lamp current of $10 \mathrm{~mA}$ was used in atomic absorption mode with an oxidizing flame (nitrous oxide-acetylene). Wavelength and slit width were 239.9 and $0.5 \mathrm{~nm}$, respectively.

Chloride was analyzed using an ion-selective electrode to check electroneutrality. The chloride ion measurements, Libelium PRO sensor probe (Libelium World, Zaragoza, Spain), which was connected to an Orion PerpHecT LogR meter model 330 (ThermoScientific, Waltham, MA, USA) was used. Chloride standards varying from 15 to $115 \mathrm{mM}$ were prepared. Starting with a known chloride solution (e.g. $0.1 \mathrm{M}$ ), calibration of the electrode was performed, and measurements were made in $\mathrm{mV}$ and converted to concentration.

For quantification of urea in the samples, QuantiChrom Urea Assay Kit (DIUR$100)$ was used. A standard urea sample solution with a concentration of $5 \mathrm{mg} / \mathrm{dL}$ was prepared. Samples, however, had to be pre-diluted to a low concentration range, which was $0.08-5 \mathrm{mg} / \mathrm{dL} .50 \mu \mathrm{L}$ of water, pre-diluted samples, and standard sample were pipetted to separate vials, $200 \mu \mathrm{L}$ of working reagent that was provided by kit was added to each vial. All samples were incubated at room temperature for $50 \mathrm{~min}$ and the absorbance were read at $430 \mathrm{~nm}$ via NanoDrop. The equation below was used to find the urea concentration:

$$
\operatorname{Urea}\left(\frac{\mathrm{mg}}{\mathrm{dL}}\right)=\frac{\mathrm{OD}_{\text {sample }}-\mathrm{OD}_{\text {blank }}}{\mathrm{OD}_{\text {standard }}-\mathrm{OD}_{\text {blank }}} * n *[\mathrm{STD}]\left(\frac{\mathrm{mg}}{\mathrm{dl}}\right)
$$

where $n$ is the dilution factor, and [STD] is the standard concentration which is equal to the value of 5 . 
A DNS (3,5-dinitrosalicylic acid) assay was used to determine the concentration of glucose. Briefly, $1 \mathrm{~g}$ of DNS was added to $100 \mathrm{~mL}$ of $2 \mathrm{~N}$ sodium hydroxide which was heated and stirred until completely dissolved. A second solution was made by combining $100 \mathrm{~g}$ of potassium sodium tartrate tetrahydrate with $250 \mathrm{~mL}$ of boiling water. These two solutions were combined while still hot. This DNS mixture was stored in a dark bottle at $4^{\circ} \mathrm{C}$. For analysis $1 \mathrm{~mL}$ of the DNS mixture was combined with $1 \mathrm{~mL}$ of the tartrate solution and diluted with di-water to a final volume of $10 \mathrm{~mL}$. The sample solutions were then boiled for $6 \mathrm{~min}$, allowed to cool on ice, and analyzed using a NanoDrop spectrophotometer at $470 \mathrm{~nm}$ and the concentration was calculated by comparison with a calibration curve constructed using glucose standards.

Bicarbonate $\left(\mathrm{HCO}_{3}{ }^{-}\right)$ion concentration of the samples was measured via an enzymatic method which can be found elsewhere ${ }^{43}$. In this method, $\mathrm{HCO}_{3}{ }^{-}$ions react with phosphoenolpyruvate (PEP) to be converted into oxaloacetate and phosphate, with phosphoenolpyruvate carboxylase (PEPC) as a catalyst (Eq. (1)). Formed oxaloacetate is catalytically reduced to malate, while nicotinamide adenine dinucleotide (NADH) is oxidated (Eq. (2)). This oxidation yields to a decrease in the absorbance of the reaction mixture, that is directly related to the bicarbonate content of the sample tested.

$$
\mathrm{PEP}+\mathrm{HCO}_{3}^{-} \stackrel{\text { PEPC }}{\longrightarrow} \text { Oxaloacetate }+\mathrm{H}_{2} \mathrm{PO}_{4}
$$

$$
\text { Oxaloacetate }+\mathrm{NADH}+\mathrm{H}^{+} \stackrel{\mathrm{MD}}{\longrightarrow} \text { Malate }+\mathrm{NAD}^{-}
$$

The assay reagent which contains malate dehydrogenase (MD), PEPC, PEP, and NADH was purchased from Beckman Coulter (Beckman Coulter, Brea, CA, USA). $500 \mu \mathrm{L}$ of the mentioned assay is pipetted into centrifuge tubes that are kept at $30{ }^{\circ} \mathrm{C}$. Initial absorbance $\left(A_{0}\right)$ of the assay is measured using a Thermo Fischer Nanodrop Spectrophotometer. Testing samples, $0.88 \mu \mathrm{L}$, are added to the tubes, vortexed and incubated for $5 \mathrm{~min}$ at $30^{\circ} \mathrm{C}$. Final absorbance of the assay-sample mixture $\left(A_{\mathrm{f}}\right)$ is recorded in triplets. The corresponding $\mathrm{CO}_{2}$ concentrations (in $\mathrm{mM}$ ) of the samples were calculated using the equation below:

$$
\mathrm{CO}_{2}(\mathrm{mM})=\frac{\left(A_{0}-A_{\mathrm{f}}\right) * V_{\mathrm{t}}}{6.22^{*} V_{\mathrm{s}}}
$$

where $V_{\mathrm{t}}$ is total volume of the sample-assay mixture, $V_{\mathrm{s}}$ is the sample volume, and 6.22 is the molar absorbtivity of NADH at $340 \mathrm{~nm}$ wavelength.

RO module function and characterization. The purpose of the RO membrane was to return water to the body to generate an artificial urine stream within the range of typical daily urine volume. One commercial RO membrane was used with the ability to have high ion rejection with the passage of water at low pressure. At a pressure of 25 psi, the ion rejections (DOW BW30) were as follows: 96\% sodium and $93 \%$ potassium over a $>24$-h period. The average flux was $\sim 41 \mathrm{~mL} / \mathrm{min}^{2}$. In order to be able to modulate the volume of water excreted by the device independent of ions and urea, an RO Sterlitech HP4750 stirred cell was utilized with a membrane area of $19.63 \mathrm{~cm}^{2}$. A solution of up to $250 \mathrm{~mL}$ was stirred at $200 \mathrm{RPM}$ at room temperature and pressurized with $\mathrm{N}_{2}$ gas at 20 psig. The RO solution was composed of $78 \mathrm{mg} / \mathrm{L}$ sodium, $6.7 \mathrm{mg} / \mathrm{L}$ potassium, $0.67 \mathrm{mg} / \mathrm{L}$ magnesium, $2.83 \mathrm{mg} / \mathrm{L}$ calcium, $78.75 \mathrm{mg} / \mathrm{L}$ chlorine, $403 \mathrm{mg} / \mathrm{L}$ urea, $53 \mathrm{mg} / \mathrm{L}$ creatinine, and $47 \mathrm{mg} / \mathrm{L}$ glucose. The RO solution used is very different than the feed solution because it takes into account what is being rejected by the other membrane components. Atomic absorption was utilized with an air-acetylene tank (40-45 psig air, 10-15 psig acetylene) to determine the rejection of ions such as sodium and potassium. For sodium, the sample was not diluted and the absorption wavelength was $330.2-330.4 \mathrm{~nm}$ with a lamp current of $5 \mathrm{~mA}$. For potassium, the sample was diluted $5 \times$ with di-water and the absorption wavelength was $769.9 \mathrm{~nm}$ with a lamp current of $6 \mathrm{~mA}$. The analysis of glucose and urea were determined using the same methods described above. Atomic absorption was utilized with an air-acetylene tank (40-45 psig air, 10-15 psig acetylene) to determine the rejection of ions such as sodium and potassium. For sodium, the sample was not diluted and the absorption wavelength was $330.2-330.4 \mathrm{~nm}$ with a lamp current of $5 \mathrm{~mA}$. For potassium, the sample was diluted $5 \times$ with di-water and the absorption wavelength was $769.9 \mathrm{~nm}$ with a lamp current of $6 \mathrm{~mA}$.

\section{Data availability}

The main data supporting the findings in this study are available within the Article and its Supplementary Information. The raw data generated in this study are available from the corresponding authors upon reasonable request.

Received: 17 September 2019; Accepted: 27 February 2020;

Published online: 27 April 2020

\section{References}

1. McCullough, K. P., Morgenstern, H., Saran, R., Herman, W. H. \& Robinson, B. M. Projecting ESRD incidence and prevalence in the United States through 2030. J. Am. Soc. Nephrol. 30, 127-135 (2019).
2. Saran, R. et al. US renal data system 2018 annual data report: epidemiology of kidney disease in the United States. Am. J. Kidney Dis. 73, A7-A8 (2019).

3. Breyer, M. D. \& Susztak, K. Developing treatments for chronic kidney disease in the 21st Century. Semin. Nephrol. 36, 436-447 (2016).

4. Gaitonde, D. Y., Cook, D. L. \& Rivera, I. M. Chronic kidney disease: detection and evaluation. Am. Fam. Physician 96, 776-783 (2017).

5. Lee, L. Y., Pham, T. A. \& Melcher, M. L. Living kidney donation: strategies to increase the donor pool. Surg. Clin. North Am. 99, 37-47 (2019).

6. Lee, M. B. \& Bargman, J. M. Survival by dialysis modality-who cares? Clin. J. Am. Soc. Nephrol. 11, 1083-1087 (2016).

7. Kaballo, M. A. et al. A comparative analysis of survival of patients on dialysis and after kidney transplantation. Clin. Kidney J. 11, 389-393 (2018).

8. Lee, S. et al. Factors affecting mortality during the waiting time for kidney transplantation: a nationwide population-based cohort study using the Korean network for organ sharing (KONOS) database. PLoS One 14, e0212748 (2019).

9. de Francisco, A. L. Future directions in therapy for chronic kidney disease. Nefrologia 30, 1-9 (2010).

10. van Gelder, M. K. et al. From portable dialysis to a bioengineered kidney. Expert Rev. Med. Devices 15, 323-336 (2018).

11. Meng, F. et al. MXene sorbents for removal of urea from dialysate: a step toward the wearable artificial kidney. ACS Nano 12, 10518-10528 (2018).

12. Yee, J. Rise of the small machines: salvation. Adv. Chronic Kidney Dis. 20, 449-451 (2013).

13. Schaeffner, E. Determining the glomerular filtration rate-an overview. J. Ren. Nutr. 27, 375-380 (2017).

14. Scott, R. P. \& Quaggin, S. E. Review series: the cell biology of renal filtration. J. Cell Biol. 209, 199-210 (2015).

15. Layton, A. T. \& Layton, H. E. A computational model of epithelial solute and water transport along a human nephron. PLoS Comput. Biol. 15, e1006108 (2019).

16. Nissenson, A. R., Ronco, C., Pergamit, G., Edelstein, M. \& Watts, R. Continuously functioning artificial nephron system: the promise of nanotechnology. Hemodial. Int. 9, 210-217 (2005).

17. Uesaka, M. [Application of nanotechnology to hemodialysis membrane] Nihon Rinsho 64, 309-315 (2006).

18. Kanno, Y. \& Miki, N. Development of a nanotechnology-based dialysis device. Contrib. Nephrol. 177, 178-183 (2012).

19. Lee, S.-J. \& Choi., B.-K. The artificial glomerulus design using diffusion in microchannels. Int. J. Precis. Eng. Man 13, 307-310 (2012).

20. Kensinger, C. et al. First implantation of silicon nanopore membrane hemofilters. ASAIO J. 62, 491-495 (2016).

21. Chevtchik, N. V. et al. Upscaling of a living membrane for bioartificial kidney device. Eur. J. Pharmacol. 790, 28-35 (2016).

22. Shankar, A. S., Hoorn, E. J., Gribnau, J., Baan, C. C. \& Hoogduijn, M. J. Current state of renal regenerative therapies. Transplantation 103, 250-261 (2019).

23. Frausto, R. F., Le, D. J. \& Aldave, A. J. Transcriptomic analysis of cultured corneal endothelial cells as a validation for their use in cell replacement therapy. Cell Transplant. 25, 1159-1176 (2016).

24. Sakolish, C. et al. Technology transfer of the microphysiological systems: a case study of the human proximal tubule tissue chip. Sci. Rep. 8, 14882 (2018).

25. Rodriguez-Boulan, E. \& Macara, I. G. Organization and execution of the epithelial polarity programme. Nat. Rev. Mol. Cell Biol. 15, 225-242 (2014).

26. Hoenig, M. P. \& Zeidel, M. L. Homeostasis, the milieu interieur, and the wisdom of the nephron. Clin. J. Am. Soc. Nephrol. 9, 1272-1281 (2014).

27. Tanaka, Y. Current density distribution and limiting current density in ionexchange membrane electrodialysis. J. Membr. Sci. 173, 179-190 (2000).

28. Potts, T. et al. The production of butanol from Jamaica Bay macro algae. Environ. Prog. Sustain. Energy 31, 29-36 (2010).

29. Massy, Z. A. \& Liabeuf, S. From old uraemic toxins to new uraemic toxins: place of 'omics'. Nephrol. Dial. Transplant. 33, iii2-iii5 (2018).

30. Kraus, M. A., Frommer, M. A., Nemas, M. \& Gutman, R. Urea-rejecting membranes and their application in the development of a miniature artificial kidney. J. Membr. Sci. 1, 115-127 (1976).

31. Ruckh, T. T. et al. Ion-switchable quantum dot forster resonance energy transfer rates in ratiometric potassium sensors. ACS Nano 10, 4020-4030 (2016).

32. Nyitrai, G. et al. Sodium selective ion channel formation in living cell membranes by polyamidoamine dendrimer. Biochim. Biophys. Acta $\mathbf{1 8 2 8}$, 1873-1880 (2013)

33. Yamaji, D. \& Takaguchi, Y. A. Novel fluorescent fluoride chemosensor based on unmodified poly(amidoamine) dendrimer. Polym. J. 41, 293-296 (2009).

34. Yuan, Z. et al. A highly ion-selective zeolite flake layer on porous membranes for flow battery applications. Angew. Chem. Int. Ed. Engl. 55, 3058-3062 (2016).

35. Hosseini, S. M., Madaeni, S. S., Heidari, A. R. \& Amirimehr, A. Preparation and characterization of ion-selective polyvinyl chloride based heterogeneous 
cation exchange membrane modified by magnetic iron-nickel oxide nanoparticles. Desal 84, 191-199 (2012).

36. Ng, C. P., Zhuang, Y., Lin, A. W. H. \& Teo, J. C. M. A fibrin-based tissueengineered renal proximal tubule for bioartificial kidney devices: development, characterization and in vitro transport study. Int. J. Tissue Eng. Article ID 319476, 1-10 (2013).

37. Umezu, M. et al. Effects of surface roughness on mechanical hemolysis. Artif. Organs 20, 575-578 (1996).

38. Wong, B. et al. Comparison of patient survival between hemodialysis and peritoneal dialysis among patients eligible for both modalities. Am. J. Kidney Dis. 71, 344-351 (2018).

39. Lee, A., Elamband, J. W. \& Darling, S. B. Membrane materials for water purification: design, development, and application. Environ. Sci. Water Res. Technol. 2, 17-42 (2016).

40. Pawlowski, S., Crespo, J. G. \& Velizarov, S. Profiled ion exchange membranes: a comprehensible review. Int. J. Mol. Sci. 20, 1-15 (2019). 165.

41. Xu, T. \& Huang, C. Electrodialysis-based separation technologies: a critical review. Separations 54, 3147-3159 (2008).

42. Arar, Ö., Yüksel, Ü., Kabay, N. \& Yüksel, M. Various applications of electrodeionization (EDI) method for water treatment-a short review. Desalination 342, 16-22 (2014).

43. Forrester, R. L., Wataji, L. J., Silverman, D. A. \& Pierre, K. J. Enzymatic method for determination of $\mathrm{CO}_{2}$ in serum. Clin. Chemistry 22, 243-245 (1976).

\section{Acknowledgements}

The authors wish to acknowledge Ashley Cox, Jessica Orton, Benjamin Drewry, Benjamin Yeldell, Jennifer Gaines, and Evin Spears for their contributions to performing experiments. The authors to acknowledge Eric Pollock for his support in conducting ICP measurements. The authors greatly appreciate the financial support from US Kidney Research Corporation as well as intellectual discussion with Roland Ludlow. I.K. is supported by funds from the NIH (DK077162), the Allan Smidt Charitable Fund, the Ralph Block Family Foundation, and the Factor Family Foundation.

\section{Author contributions}

J.A.H., C.N.H., and I.K. designed the study; S.P., G.M., E.P., J.M., L.S., S.S., and C.A. performed experiments and collected the data; J.A.H., C.N.H., and I.K. analyzed the data;
J.A.H., C.N.H., and I.K. gave technical support and conceptual device; J.A.H., C.N.H., and I.K. wrote the manuscript.

\section{Competing interests}

The authors declare the following competing interests. I.K. is scientific and medical advisor on the board of US Kidney Research Corporation. I.K. has received shares from U.S. Kidney Research Corporation. US Kidney Research Corporation provided funding for the research to C.N.H., J.A.H., S.P., G.M., E.P., J.M., L.S., S.S., and C.A.

\section{Additional information}

Supplementary information is available for this paper at https://doi.org/10.1038/s43246020-0016-3.

Correspondence and requests for materials should be addressed to I.K.

Reprints and permission information is available at http://www.nature.com/reprints

Publisher's note Springer Nature remains neutral with regard to jurisdictional claims in published maps and institutional affiliations.

(c) (i) Open Access This article is licensed under a Creative Commons Attribution 4.0 International License, which permits use, sharing, adaptation, distribution and reproduction in any medium or format, as long as you give appropriate credit to the original author(s) and the source, provide a link to the Creative Commons license, and indicate if changes were made. The images or other third party material in this article are included in the article's Creative Commons license, unless indicated otherwise in a credit line to the material. If material is not included in the article's Creative Commons license and your intended use is not permitted by statutory regulation or exceeds the permitted use, you will need to obtain permission directly from the copyright holder. To view a copy of this license, visit http://creativecommons.org/ licenses/by/4.0/.

(C) The Author(s) 2020 